\title{
Pressure effect upon the dielectric response of KDP family crystals
}

\author{
I.V.Stasyuk, R.R.Levitskii, A.P.Moina \\ Institute for Condensed Matter Physics \\ of the National Academy of Sciences of Ukraine, \\ 1 Svientsitskii Str., 79011 Lviv, Ukraine
}

Received January 14, 1999

\begin{abstract}
Within the four-particle cluster approximation for the proton ordering model, we study the effects of external pressures (which do not lower the crystals' symmetry) upon static and dynamic dielectric properties of deuterated ferroelectrics and antiferroelectrics of $\mathrm{KH}_{2} \mathrm{PO}_{4}$ family. The theory provides a satisfactory description of the available experimental data. The importance of the pressure induced changes in the D-site distance in the dielectric response of the hydrogen bonded crystals is shown.
\end{abstract}

Key words: KDP, pressure, spontaneous polarization, dielectric permittivity

PACS: $77.84 . F a$

In spite of serious doubts regarding the validity of the proton ordering model raised by Raman scattering experiments [1,2], this model still remains the most elaborated and widely used approach to the description of the phase transitions in the KDP family crystals. According to the proton ordering model, these phase transitions are triggered by ordering of protons on hydrogen bonds, which leads to displacements of heavy ions, hence a spontaneous polarization arises. Therefore, the hydrogen subsystem and, particularly, the geometry of hydrogen bonds seem to play an important role in dipole moment formation in these H-bonded crystals.

High pressure studies, being the only means of continually varying the parameters of hydrogen bonds, are the best tool of exploring the role of hydrogen bonds subsystem as well as the H-bond geometry in the physics involved. Amongst the examples of important insights into the problem provided by high pressure studies, there is the universality of the transition temperature vs. H-site dependence [3] observed by means of neutron scattering techniques in $\mathrm{KDP}, \mathrm{KD}_{2} \mathrm{PO}_{4}, \mathrm{NH}_{4} \mathrm{H}_{2} \mathrm{PO}_{4}$, and $\mathrm{ND}_{4} \mathrm{D}_{2} \mathrm{PO}_{4}$ strained by hydrostatic pressure. Our theoretical calculations have shown [4-6] that this universality is obeyed under the hydrostatic pressure by all six deuterated crystals of $\mathrm{KH}_{2} \mathrm{PO}_{4}$ family $\mathrm{MeD}_{2} \mathrm{XO}_{4}\left(\mathrm{Me}=\mathrm{K}, \mathrm{Rb}, \mathrm{ND}_{4}, \mathrm{X}=\mathrm{P}, \mathrm{As}\right)$ with a three dimensional network of hydrogen bonds as well as by $\mathrm{K}\left(\mathrm{H}_{0.13} \mathrm{D}_{0.87}\right){ }_{2} \mathrm{PO}_{4}$ 
under the uniaxial pressure $p=-\sigma_{3}$.

Usually, the pressure effects in KDP family crystals are explained within the proton ordering model in the mean field approximation assuming a decrease in the parameter of hydrogen-hydrogen interaction $J$ and an increase in the tunneling integral (see $[7,8]$ ). A more adequate description of the phenomena has been obtained in the four particle cluster approximation by Blinc [9] and Torstveit [10]. The derivatives of transition temperature, spontaneous polarization, and Curie constant with respect to hydrostatic pressure for KDP and DKDP were successfully described. However, since then a lot of new experimental data on the pressure effects have become available for other crystals of KDP family [11-16] and for uniaxial pressures [17].

In our recent papers [4-6,20-22] we developed a unified approach allowing us to consistently describe the effect of external pressure upon several ferroelectric and antiferroelectric crystals of KDP family. In this paper we present the results of the studies of hydrostatic and uniaxial pressure effects upon the static and the dynamic dielectric response of the considered crystals within this approach.

\section{The model}

We consider a deuteron subsystem of a strained by hydrostatic or uniaxial $p=$ $-\sigma_{3}$ pressure ferroelectric $(\mathrm{FE})$ or an antiferroelectric (AFE) crystal of $\mathrm{KH}_{2} \mathrm{PO}_{4}$ family with a general formula $\mathrm{MeD}_{2} \mathrm{XO}_{4}\left(\mathrm{Me}=\mathrm{K}, \mathrm{Rb}, \mathrm{ND}_{4}, \mathrm{X}=\mathrm{P}, \mathrm{As}\right)$, In our calculations we use the recently developed model of deuterated strained crystals of $\mathrm{KH}_{2} \mathrm{PO}_{4}$ type [4-6,20-22].

Calculations are performed within the four-particle cluster approximation which allows one to take adequately into account the strong short-range correlations between deuterons. The four-particle cluster Hamiltonian of the system has the following conventional form:

$$
\begin{aligned}
H_{q}= & V\left[\frac{\sigma_{q 1}}{2} \frac{\sigma_{q 2}}{2}+\frac{\sigma_{q 2}}{2} \frac{\sigma_{q 3}}{2}+\frac{\sigma_{q 3}}{2} \frac{\sigma_{q 4}}{2}+\frac{\sigma_{q 4}}{2} \frac{\sigma_{q 1}}{2}\right] \\
& +U\left[\frac{\sigma_{q 1}}{2} \frac{\sigma_{q 3}}{2}+\frac{\sigma_{q 2}}{2} \frac{\sigma_{q 4}}{2}\right]+\Phi \frac{\sigma_{q 1}}{2} \frac{\sigma_{q 2}}{2} \frac{\sigma_{q 3}}{2} \frac{\sigma_{q 4}}{2}-\sum_{f=1}^{4} \frac{z_{q f}^{i}}{\beta} \frac{\sigma_{q f}}{2},
\end{aligned}
$$

where two eigenvalues of the Ising spin $\sigma_{q f}= \pm 1$ are assigned to two deuteron sites on the $f$-th bond in the $q$-th cell, tunneling being neglected.

Constants $V, U$, and $\Phi$, describing the short-range correlations between deuterons, are given by the following functions of Slater energies $\varepsilon, w$, and $w_{1}$

$$
\begin{array}{ll}
\text { FE }: & V=-\frac{w_{1}}{2}, \quad U=-\varepsilon+\frac{w_{1}}{2}, \quad \Phi=4 \varepsilon-8 w+2 w_{1} ; \\
\text { AFE }: & V=\frac{\varepsilon-w_{1}}{2}, \quad U=\frac{\varepsilon+w_{1}}{2}, \quad \Phi=2 \varepsilon-8 w+2 w_{1} .
\end{array}
$$

The fields $z_{q f}^{i}$ include effective cluster fields $\Delta_{q f}^{i}$, external electric fields $E_{i}$, and the long-range interactions (dipole-dipole and indirect via lattice vibrations) taken into 
account in the mean field approximation

$$
z_{q f}^{i}=\beta\left[-\Delta_{q f}^{i}+\sum_{q^{\prime} f^{\prime}} J_{f f^{\prime}}\left(q q^{\prime}\right) \frac{\left\langle\sigma_{q^{\prime} f^{\prime}}\right\rangle}{2}+\mu_{q f}^{i} E_{i}\right] .
$$

A variation with the pressure of the Slater energies $\varepsilon, w$, and $w_{1}$ and the components of the long-range interaction matrix $J_{f f^{\prime}}\left(q q^{\prime}\right)$ is modelled in the following way $[4,5]$

$$
\begin{gathered}
\varepsilon=\varepsilon^{0}\left[1-\frac{2}{S} \frac{\delta_{1}}{\delta_{0}} \sum_{j=1}^{3} \varepsilon_{j}\right]+\sum_{i=1}^{3} \delta_{1 i} \varepsilon_{i}, \quad w=w^{0}\left[1-\frac{2}{S} \frac{\delta_{1}}{\delta_{0}} \sum_{j=1}^{3} \varepsilon_{j}\right]+\sum_{i=1}^{3} \delta_{2 i} \varepsilon_{i} \\
w_{1}=w_{1}^{0}\left[1-\frac{2}{S} \frac{\delta_{1}}{\delta_{0}} \sum_{j=1}^{3} \varepsilon_{j}\right]+\sum_{i=1}^{3} \delta_{3 i} \varepsilon_{i} \\
J_{f f^{\prime}}\left(q q^{\prime}\right)=J_{f f^{\prime}}^{(0)}\left(q q^{\prime}\right)\left[1-\frac{2}{S} \frac{\delta_{1}}{\delta_{0}} \sum_{j=1}^{3} \varepsilon_{j}\right]+\sum_{j=1}^{3} \psi_{f f^{\prime}}^{j}\left(q q^{\prime}\right) \varepsilon_{j} .
\end{gathered}
$$

Here the contributions of both the lattice strains (via $\psi_{f f^{\prime}}^{i}\left(q q^{\prime}\right)$ and $\left.\delta_{i j}\right)$ and of the pressure-induced changes in the D-site distance (via $\delta_{1} / \delta_{0}$ are taken into account. $S=\sum_{i j} S_{i j}^{(0)}$ and $S=\sum_{j} S_{3 j}^{(0)}$ for hydrostatic and uniaxial $p=-\sigma_{3}$ pressures, respectively; $S_{i j}^{(0)}$ are elastic compliances, and

$$
\delta=\delta_{0}+\delta_{1} p
$$

is an assumed pressure dependence of the D-site distance $\left.{ }^{1}\right)$.

We shall consider only a longitudinal electric field $E_{3}$ in ferroelectric crystals and a transverse electric field $E_{1}$ in antiferroelectric crystals. It brings about the following symmetry of the quasispin mean values, effective fields $z_{q f}^{i}$, and effective dipole moments $\mu_{q f}^{i}$ :

$$
\begin{aligned}
& \mathrm{FE}: \quad \eta^{f} \equiv\left\langle\sigma_{q 1}\right\rangle=\left\langle\sigma_{q 2}\right\rangle=\left\langle\sigma_{q 3}\right\rangle=\left\langle\sigma_{q 4}\right\rangle \\
& \quad \mu_{3} \equiv \mu_{q 1}^{3}=\mu_{q 2}^{3}=\mu_{q 3}^{3}=\mu_{q 4}^{3} ; \quad z^{f} \equiv z_{q 1}^{3}=z_{q 2}^{3}=z_{q 3}^{3}=z_{q 4}^{3} ; \\
& \mathrm{AFE}: \quad \eta_{q 13}^{a} \equiv-\left\langle\sigma_{q 1}\right\rangle=\left\langle\sigma_{q 3}\right\rangle, \quad \eta_{q 24}^{a}=-\left\langle\sigma_{q 4}\right\rangle=\left\langle\sigma_{q 2}\right\rangle, \\
& \quad \mu_{1} \equiv-\mu_{q 1}^{1}=\mu_{q 3}^{1}, \quad \mu_{q 2}^{1}=\mu_{q 4}^{1}=0, \quad z_{q 13}^{a} \equiv-z_{q 1}^{1}=z_{q 3}^{1}, \quad z_{q 24}^{a} \equiv z_{q 2}^{1}=-z_{q 4}^{1} .
\end{aligned}
$$

The order parameter (mean value of the quasispin) of a ferroelectric crystal is uniform, whereas that of an antiferroelectric crystal should be presented as the sum of a spontaneous modulated part and of a field-induced uniform part:

$$
\begin{aligned}
& \eta_{q f}^{a}=\eta_{f}^{a} \mathrm{e}^{i \boldsymbol{k}_{Z} \boldsymbol{R}_{q}}+\eta_{f E}^{a}, \quad \eta^{a}=-\eta_{1}^{a}=\eta_{2}^{a}=\eta_{3}^{a}=-\eta_{4}^{a}, \\
& \eta_{13 E}^{a} \equiv \eta_{3 E}^{a}=-\eta_{1 E}^{a}, \quad \eta_{24 E}^{a} \equiv \eta_{2 E}^{a}=-\eta_{4 E}^{a} .
\end{aligned}
$$

\footnotetext{
${ }^{1}$ according to $[18,19]$, the variation of $\delta$ with hydrostatic pressure in $\mathrm{KD}_{2} \mathrm{PO}_{4}$ is linear indeed, except that $\delta_{0}$ and $\delta_{1}$ are temperature dependent
} 
Here $\boldsymbol{k}_{Z}=\left(\boldsymbol{b}_{1}+\boldsymbol{b}_{2}+\boldsymbol{b}_{3}\right) / 2, \boldsymbol{b}_{1}, \boldsymbol{b}_{2}, \boldsymbol{b}_{3}$ are the basic vectors of the reciprocal lattice; the factor $\mathrm{e}^{i \boldsymbol{k}_{Z} \boldsymbol{R}_{q}}= \pm 1$ denotes two sublattices of the antiferroelectrics.

Excluding the effective fields $\Delta$ from $z$ by using the self-consistency condition, which states that the quasispins mean values calculated with the four-particle Hamiltonian (1) and with the one-particle Hamiltonian

$$
H_{q f}^{(1)}=-\frac{\bar{z}_{q f}^{i}}{\beta} \frac{\sigma_{q f}}{2}, \quad \bar{z}_{q f}^{i}=z_{q f}^{i}-\beta \Delta_{q f}^{i}
$$

should coincide, we obtain

$$
\begin{aligned}
\mathrm{FE}: & z^{f}=\frac{1}{2} \ln \frac{1+\eta^{f}}{1-\eta^{f}}+\beta \nu_{c}(0) \eta^{f}+\frac{\beta \mu_{3} E_{3}}{2} \\
\mathrm{AFE}: & z_{q 13}^{a}=\frac{1}{2} \ln \frac{1+\eta_{q 13}^{a}}{1-\eta_{q 13}^{a}}+\beta\left[\nu_{a}\left(\boldsymbol{k}_{Z}\right) \eta^{a} \mathrm{e}^{i \boldsymbol{k}_{Z} \boldsymbol{R}_{q}}+\nu_{a}(0) \eta_{13 E}^{a}+\frac{\mu_{1} E_{1}}{2}\right], \\
z_{q 24}^{a} & =\frac{1}{2} \ln \frac{1+\eta_{q 24}^{a}}{1-\eta_{q 24}^{a}}+\beta\left[\nu_{a}\left(\boldsymbol{k}_{Z}\right) \eta^{a} \mathrm{e}^{i \boldsymbol{k}_{Z} \boldsymbol{R}_{q}}+\nu_{a}(0) \eta_{24 E}^{a}\right]
\end{aligned}
$$

with the eigenvalues of Fourier transforms of the long-range interaction matrices given by

$$
\begin{aligned}
& \nu_{c}(0)=\frac{1}{4}\left(J_{11}(0)+2 J_{12}(0)+J_{13}(0)\right)=\nu_{c}^{0}(0)\left[1-\frac{2}{S} \frac{\delta_{1}}{\delta_{0}} \sum_{j=1}^{3} \varepsilon_{j}\right]+\sum_{i=1}^{3} \psi_{c i}(0) \varepsilon_{i}, \\
& \nu_{a}(\boldsymbol{k})=\frac{1}{4}\left(J_{11}(\boldsymbol{k})-J_{13}(\boldsymbol{k})\right)=\nu_{a}^{0}(\boldsymbol{k})\left[1-\frac{2}{S} \frac{\delta_{1}}{\delta_{0}} \sum_{j=1}^{3} \varepsilon_{j}\right]+\sum_{i=1}^{3} \psi_{a i}(\boldsymbol{k}) \varepsilon_{i} .
\end{aligned}
$$

The order parameter and lattice strains are to be found from

$$
\eta^{f, a}=\frac{1}{D^{f, a}}\left(\sinh 2 z^{f, a}+2 b \sinh z^{f, a}\right), \quad-p_{i}=\sum_{j=1}^{3} c_{i j} \varepsilon_{j},
$$

electrostriction is neglected; $p_{i}=(p, p, p)$ and $p_{i}=(0,0, p)$ for the hydrostatic and uniaxial pressure $p=-\sigma_{3}$, respectively; $c_{i j}$ are the crystal elastic constants; the first equation in (4) was obtained by minimization of thermodynamic potential $[4,5]$ with respect to $\eta$;

$$
\begin{aligned}
& D^{f}=\cosh 2 z^{f}+4 b \cosh z^{f}+2 a+d, \quad D^{a}=\cosh 2 z^{a}+4 b \cosh z^{a}+a+d+1, \\
& a=\exp (-\beta \varepsilon), \quad b=\exp (-\beta w), \quad d=\exp \left(-\beta w_{1}\right) .
\end{aligned}
$$

\section{Dielectric characteristics}

It is assumed that the polarization of the crystal, triggered by deuteron ordering, is related to the mean values of quasispins as

$$
P_{i}=\sum_{f} \frac{\mu_{q f}^{i}}{v} \frac{\left\langle\sigma_{q f}\right\rangle}{2}
$$


with summation going over all sites of a unit cell (summation over a primitive cell would give a sublattice polarization). Taking into account the system symmetry, we can write that

$$
\text { FE : } \quad P_{3}=\frac{4 \mu_{3}}{v} \eta^{f}, \quad \text { AFE }: \quad P_{1}=\frac{2 \mu_{1}}{v} \eta_{13 E}^{a} ;
$$

$v$ is the unit cell volume. It is the variation of the effective dipole moments $\mu_{i}$ with pressure that governs the pressure dependences of dielectric characteristics of the crystals. Determination of this variation will be deferred till the next section.

Differentiation of (5) with respect to $E_{3}$ or $E_{1}$ at $\partial \varepsilon_{i} / \partial E_{j}=0$ yields the static dielectric permittivities of clamped crystals.

$$
\begin{array}{ll}
\mathrm{FE}: & \varepsilon_{3}^{f}(T, p)=\varepsilon_{3 \infty}^{f}+4 \pi \frac{\beta \mu_{3}^{2}}{v} \frac{4 \varkappa_{3}^{f}}{D^{f}-2 \varphi_{3}^{f} \varkappa_{3}^{f}}, \\
\mathrm{AFE}: & \varepsilon_{1}^{a}(T, p)=\varepsilon_{1 \infty}^{a}+4 \pi \frac{\beta \mu_{1}^{2}}{2 v}\left[\frac{2 \varkappa_{1}^{a}}{D^{a}-2 \varkappa_{1}^{a} \varphi_{1}^{a}}+\frac{2 \varkappa_{2}^{a}}{D^{a}-2 \varkappa_{2}^{a} \varphi_{1}^{a}}\right] ;
\end{array}
$$

$\varepsilon_{i \infty}$ are high-frequency contributions to the permittivities, and

$$
\begin{aligned}
\varkappa_{3}^{f} & =\cosh 2 z^{f}+b \cosh z^{f}-\left[\eta^{f}\right]^{2} D^{f}, \\
\varkappa_{1}^{a} & =1+b \cosh z^{a}, \varkappa_{2}^{a}=\cosh 2 z^{a}+b \cosh z^{a}-\left[\eta^{a}\right]^{2} D^{a} ; \\
\varphi_{3}^{f} & =\frac{1}{1-\left[\eta^{f}\right]^{2}}+\beta \nu_{c}(0), \quad \varphi_{1}^{a}=\frac{1}{1-\left[\eta^{a}\right]^{2}}+\beta \nu_{a}(0) .
\end{aligned}
$$

At an ambient pressure above $T_{\mathrm{N} 0}$ and in the limit $w \rightarrow \infty$ and $w_{1} \rightarrow \infty$, the obtained expression for $\varepsilon_{1}^{a}$ coincides with that of Havlin [23].

Permittivities of mechanically free and clamped crystals are related by

$$
\tilde{\varepsilon}_{3}^{f}(p, T)=\varepsilon_{3}^{f}(p, T)+4 \pi\left(\sum_{i=1}^{3} e_{3 i} d_{3 i}+e_{36} d_{36}\right), \quad \tilde{\varepsilon}_{1}^{a}(p, T)=\varepsilon_{1}^{a}(p, T)+4 \pi d_{14}^{2} c_{44},
$$

$d_{i j}$ and $e_{i j}$ are crystal piezomodules.

Let us now consider the dynamic dielectric characteristics of the studied crystals. Starting from the Glauber master equation [24] which for the the expectation values of the products of spins can be written as

$$
-\alpha \frac{\mathrm{d}}{\mathrm{d} t}\left\langle\prod_{f} \sigma_{q f}\right\rangle=\sum_{f}\left\langle\prod_{f^{\prime}} \sigma_{q f^{\prime}}\left[1-\sigma_{q f} \tanh \frac{1}{2} \beta \mathcal{E}_{q f}\right]\right\rangle
$$

taking into account the symmetry of the products $\left\langle\prod_{f} \sigma_{q f}\right\rangle$ in the considered electric fields $E_{3}(t)$ in ferroelectrics and $E_{1}(t)$ in antiferroelectrics, we obtain systems of differential equations (four equations for ferroelectrics and ten equations for antiferroelectrics) $[20,21]$. Those systems are solved under the assumptions: i) the electric fields $E_{j}(t)=E_{j 0} \exp (-\mathrm{i} \omega t)$ are weak and cause only small departures from thermal equilibrium, and ii) the strains $\varepsilon_{i}$ are time independent. At frequencies of the external electric field above the mechanical resonance frequency of a crystal $\left(\sim 10^{6}\right)$, the 
piezoelectric deformation is not able to instantly follow the periodic changes in the applied electric field [25]. Therefore, due to inertia effects, at the considered in this work frequencies $\left(10^{9} \div 10^{12} \mathrm{~Hz}\right)$, a susceptibility of effectively clamped crystals is measured, and neglecting time fluctuations of strains is justified.

Solving the obtained systems of differential equations, we find that above the transition point there are two relaxational modes in both longitudinal dielectric relaxation in the ferroelectrics and transverse relaxation in the antiferroelectrics. The corresponding components of dynamic dielectric permittivity tensors contain two Debye contributions

$$
\varepsilon_{i}^{\prime+}(\omega, T, p)=\varepsilon_{\mathrm{i} \infty}+4 \pi \sum_{j=1}^{2} \frac{\chi_{i}^{i}}{1+\left(\omega \tau_{j}^{i}\right)^{2}}, \quad \varepsilon_{3}^{\prime \prime+}(\omega, T, p)=4 \pi \sum_{j=1}^{2} \frac{\omega \tau_{j}^{i} \chi_{j}^{i}}{1+\left(\omega \tau_{j}^{i}\right)^{2}} .
$$

Somewhat cumbersome expressions for the relaxation times $\tau_{j}^{i}$ and coefficients $\chi_{j}^{i}$ are given elsewhere $[21,27,20]$.

\section{The fitting procedure}

Values of the theory parameters were chosen such that the best fit to available experimental data for the pressure dependences of transition temperatures was obtained for each of the considered $\mathrm{MeD}_{2} \mathrm{XO}_{4}\left(\mathrm{Me}=\mathrm{K}, \mathrm{Rb}, \mathrm{ND}_{4}, \mathrm{X}=\mathrm{P}, \mathrm{As}\right)$ crystals. A detailed description of the fitting procedure and setting the theory parameters is given elsewhere $[4,5]$.

It should be emphasized here that the most important parameter in determining the pressure dependence of the transition temperature is the ratio $\delta_{1} / \delta_{0}$ which is the rate of the pressure changes in the D-site distance. For all crystals and pressures, we treat $\delta_{1} / \delta_{0}$ as a free parameter. The unexpected outcome of the fitting process is the prediction that since the uniaxial pressure $p=-\sigma_{3}$ decreases the transition temperature in $\mathrm{KD}_{2} \mathrm{PO}_{4}$ [17], it must shorten the hydrogen bonds and D-site distances $\left(\delta_{1} / \delta_{0}<0\right)$; one would rather expect the pressure applied along the $c$-axis to expand the D-bonds lying in the $a b$-plane. One of the possible explanations of this shortening is that $p=-\sigma_{3}$ pressure flattens $\mathrm{PO}_{4}$ tetrahedra along the $c$-axis, thereby enlarging their projection on the $a b$-plane and reducing the distances between oxygens of different $\mathrm{PO}_{4}$ groups. Another reason for such elongation could be a rotation of $\mathrm{PO}_{4}$ tetrahedra around the $c$-axis in a direction opposite to the direction in which they rotate under hydrostatic pressure $[18,19]$. However, these conjectures should await an experimental verification.

The values of the theory parameters yielding the best fit of theoretical dependences of transition temperature on pressure to experimental data are given in table 1 . Values of deuteration $x$ for $\mathrm{K}\left(\mathrm{H}_{1-x} \mathrm{D}_{x}\right)_{2} \mathrm{PO}_{4}$ crystals are nominal. For all crystals $\delta_{i j}=0, w_{1} \rightarrow \infty$.

These values of the theory parameters give the universal transition temperature vs. D-site distance $\delta$ dependence for six deuterated crystals of KDP family [4-6], which again manifests the importance of the D-site distance $\delta$ in the phase transitions 
in these hydrogen bonded crystals. Hereinafter we shall show that $\delta$ palys a crucial role in the dielectric response of these crystals as well, since the ratio $\delta_{1} / \delta_{0}$ also determines the pressure dependence of their dielectric characteristics.

The slopes $\partial \mu_{i} / \partial p$ which govern the pressure dependence of the spontaneous polarization and dielectric permittivities of the studied crystals can be determined without introducing any extra fitting parameter into the theory based on the following speculations.

It is believed that the deuteron ordering in the system results in displacements of heavy ions and electron density which contribute to crystal polarization. Since, when ordered, a deuteron shifts from its central position on a hydrogen bond to the off-central position by a distance of $\delta / 2$, it seems reasonable to assume that the heavy ions displacements are also proportional to $\delta$. This idea was used in the previous theories [10]. In the present work we also assume that $\mu_{i}$ is proportional to the corresponding lattice constant $a_{i}$, reflecting an intuitively perceived fact that the larger the molecule is the greater the dipole moment arises in it. This yields

$$
\frac{1}{\mu_{i}^{0}} \frac{\partial \mu_{i}}{\partial p}=\frac{\delta_{1}}{\delta_{0}}+\frac{\varepsilon_{i}}{p}
$$

The antiferroelectric crystals of the KDP family are characterized by the large values of transverse effective dipole moments of the unit cell. A strong variation with pressure of the permittivity $\varepsilon_{1}(T, p)$ of $\mathrm{ND}_{4} \mathrm{D}_{2} \mathrm{AsO}_{4}[16]$ is described under the assumption that, in addition to ion shifts due to the deuteron ordering, there should be some other factor in the mechanism of dipole moment formation in these crystals. We assume that there exists an interaction between large dipole moments of unit cells (we call this a mutual polarization), which changes their magnitude and the character of their pressure dependence. Within a simple model $[4,5,22]$ in which a unit cell $i$ is assigned a dipole moment $\boldsymbol{d}_{i}$ (the size of the dipoles is much smaller than the distance between them), supposing that the magnitude of the dipole moment is proportional to a complete electric field acting on it: the external field $\boldsymbol{E}_{0}$ plus the internal field created by other dipoles of the crystal

$$
\boldsymbol{d}_{i}=\hat{\alpha}\left(\boldsymbol{E}_{0}+\sum_{j} \frac{3\left(\boldsymbol{n}_{i j} \boldsymbol{d}_{j}\right) \boldsymbol{n}_{i j}-\boldsymbol{d}_{j}}{R_{i j}^{3}}\right),
$$

(where $\hat{\alpha}$ is the polarizability tensor, $\boldsymbol{n}_{i j}$ is the unit vector directed from the dipole $\boldsymbol{d}_{j}$ to $\boldsymbol{d}_{i}$, and $R_{i j}$ is the distance between the dipoles), if all dipoles are directed along the external field, and the magnitudes of the dipoles are all the same, we obtain (see $[4,5,22])$

$$
\frac{1}{\mu_{1}^{2}} \frac{\partial \mu_{1}^{2}}{\partial p}=\frac{1}{\alpha_{1}} \frac{\partial \alpha_{1}}{\partial p}\left[1+\tilde{\chi}_{1} K_{1} \mu_{1}^{2}\right]+\tilde{\chi}_{1} \mu_{1}^{2} \frac{\partial K_{1}}{\partial p}
$$

where

$$
\tilde{\chi}_{1}=\frac{1}{2}\left[\frac{2 \varkappa_{1}^{a}}{D^{a}-2 \varkappa_{1}^{a} \varphi_{1}^{a}}+\frac{2 \varkappa_{2}^{a}}{D^{a}-2 \varkappa_{2}^{a} \varphi_{1}^{a}}\right],
$$


Table 1. The theory parameters for the considered crystals.

$\nu^{0}=\nu_{c}^{0}(0)$ and $\psi_{i}=\psi_{c i}(0)$ for ferroelectrics; $\nu^{0}=\nu_{a}^{0}\left(\boldsymbol{k}_{Z}\right)$ and $\psi_{i}=\psi_{a i}\left(\boldsymbol{k}_{Z}\right)$ for antiferroelectrics. $\psi_{i}^{-}$and $\psi_{i}^{+}$are the values of the deformation potentials below and above transition point, respectively.

$f^{0}=\left(\mu_{3}^{0}\right)^{2} / v$ for ferroelectrics and $f^{0}=\left(\mu_{1}^{0}\right)^{2} / v$ for antiferroelectrics.

Values of $\partial T_{\mathrm{C}} / \partial p$ and $\delta_{1} / \delta_{0}$ for this crystal correspond to uniaxial pressure $p=$ $-\sigma_{3}$.

Values of deuteration $x$ for $\mathrm{K}\left(\mathrm{H}_{1-x} \mathrm{D}_{x}\right)_{2} \mathrm{PO}_{4}$ crystals are nominal;

difference between elastic constants of $\mathrm{K}\left(\mathrm{H}_{0.13} \mathrm{D}_{0.87}\right){ }_{2} \mathrm{PO}_{4}$ and $\mathrm{K}\left(\mathrm{H}_{0.16} \mathrm{D}_{0.84}\right)_{2} \mathrm{PO}_{4}$ crystals with $T_{\mathrm{C} 0}=210 \mathrm{~K}$ and $T_{\mathrm{C} 0}=208 \mathrm{~K}$, respectively, is neglected.

\begin{tabular}{|c|c|c|c|c|c|c|c|}
\hline crystal & $\begin{array}{l}T_{\mathrm{C} 0} \\
(\mathrm{~K})\end{array}$ & $\begin{array}{c}\partial T_{\mathrm{C}} / \partial p \\
(\mathrm{~K} / \mathrm{kbar})\end{array}$ & $\begin{array}{c}\delta_{1} / \delta_{0} \\
\left(10^{-3} \mathrm{kbar}^{-1}\right)\end{array}$ & $\psi_{1}^{-} \quad \psi_{2}^{-}$ & $\begin{array}{c}\psi_{3}^{-} \\
(\mathrm{K})\end{array}$ & $\psi_{1}^{+}$ & $\psi_{3}^{+}$ \\
\hline $\mathrm{KD}_{2} \mathrm{PO}_{4}$ & 220 & $-2.0[12]$ & -6.4 & 130110 & -560 & 120 & -560 \\
\hline $\mathrm{KD}_{2} \mathrm{PO}_{4}$ & 220 & $-2.5[11]$ & -7.4 & 130110 & -560 & 120 & -560 \\
\hline $\mathrm{K}\left(\mathrm{H}_{0.13} \mathrm{D}_{0.87}\right){ }_{2} \mathrm{PO}_{4}$ & 210 & $-12.5[17]$ & -35.0 & 120100 & -545 & 110 & -545 \\
\hline $\mathrm{K}\left(\mathrm{H}_{0.16} \mathrm{D}_{0.84}\right){ }_{2} \mathrm{PO}_{4}$ & 208 & $-3.0[11]$ & -9.3 & 11090 & -545 & 100 & -545 \\
\hline $\mathrm{RbD}_{2} \mathrm{PO}_{4}$ & 207.3 & $-3.7[14]$ & -10.7 & 110 & -450 & 100 & -450 \\
\hline $\mathrm{ND}_{4} \mathrm{D}_{2} \mathrm{PO}_{4}$ & 235.0 & $-1.4[26]$ & -4.9 & 310290 & -500 & 290 & -560 \\
\hline $\mathrm{ND}_{4} \mathrm{D}_{2} \mathrm{AsO}_{4}$ & 286.3 & $-1.4[16]$ & -4.4 & 380360 & -680 & 370 & -680 \\
\hline
\end{tabular}

\begin{tabular}{|c|c|c|c|c|c|c|}
\hline crystal & $\begin{array}{l}T_{\mathrm{C} 0} \\
(\mathrm{~K})\end{array}$ & & $\begin{array}{l}w^{0} \\
(\mathrm{~K})\end{array}$ & $\nu^{0}$ & $\begin{array}{c}f^{0} \\
(\mathrm{~K})\end{array}$ & $\begin{array}{c}\mu_{3}^{0-} / v \\
\left(\mu \mathrm{C} / \mathrm{cm}^{2}\right.\end{array}$ \\
\hline $\mathrm{KD}_{2} \mathrm{PO}_{4}$ & 220 & 92.0 & 830 & 38.0 & 76 & 1.55 \\
\hline $\mathrm{K}\left(\mathrm{H}_{0.13} \mathrm{D}_{0.87}\right)_{2} \mathrm{PO}_{4}$ & 210 & 87.6 & 785 & 37.05 & & 1.49 \\
\hline $\mathrm{K}\left(\mathrm{H}_{0.16} \mathrm{D}_{0.84}\right)_{2} \mathrm{PO}_{4}$ & 208 & 87.6 & 785 & 36.0 & & \\
\hline $\mathrm{RbD}_{2} \mathrm{PO}_{4}$ & 207.3 & 95.9 & 828 & 29.9 & 13.5 & \\
\hline $\mathrm{ND}_{4} \mathrm{D}_{2} \mathrm{PO}_{4}$ & 235.0 & 77.0 & 709 & 85.75 & 837 & \\
\hline $\mathrm{ND}_{4} \mathrm{D}_{2} \mathrm{AsO}_{4}$ & 286.3 & 105.0 & 810 & 103.65 & 1400 & \\
\hline
\end{tabular}

\begin{tabular}{|l|cccc|cccccc|}
\hline crystal & $c_{11}^{+}$ & $c_{12}^{+}$ & $c_{13}^{+}$ & $c_{33}^{+}$ & $c_{11}^{-}$ & $c_{12}^{-}$ & $c_{13}^{-}$ & $c_{22}^{-}$ & $c_{23}^{-}$ & $c_{33}^{-}$ \\
& \multicolumn{10}{|c}{$\left(10^{5}\right.$ bar $)$} \\
\hline $\mathrm{KD}_{2} \mathrm{PO}_{4}$ & 6.14 & -0.71 & 1.05 & 4.82 & 6.14 & -0.71 & 1.0 & 6.14 & 1.1 & 4.3 \\
$\mathrm{~K}_{\left(\mathrm{H}_{0.13} \mathrm{D}_{0.87}\right)_{2} \mathrm{PO}_{4}}$ & 6.93 & -0.78 & 1.22 & 5.45 & 6.8 & -0.78 & 1.0 & 6.99 & 1.0 & 5.3 \\
$\mathrm{RbD}_{2} \mathrm{PO}_{4}$ & 6.85 & -0.1 & 1.3 & 5.2 & 6.85 & -0.1 & 1.3 & 6.85 & 1.2 & 5.2 \\
$\mathrm{ND}_{4} \mathrm{D}_{2} \mathrm{PO}_{4}$ & 6.28 & 0.39 & 1.9 & 3.25 & 6.2 & 0.39 & 1.6 & 6.28 & 1.59 & 3.25 \\
$\mathrm{ND}_{4} \mathrm{D}_{2} \mathrm{AsO}_{4}$ & 6.4 & 0.9 & 2.4 & 3.85 & 6.4 & 0.9 & 2.4 & 6.3 & 2.2 & 3.85 \\
\hline
\end{tabular}




$$
\begin{aligned}
& K_{1}=\frac{r_{1}}{R^{5}}, \quad \frac{\partial K_{1}}{\partial p} p=\sum_{n_{1} n_{2} n_{3}} \frac{a^{2} n_{1}^{2}\left(4 R^{2}-5 r_{1}\right) \varepsilon_{1}-\left(2 R^{2}+5 r_{1}\right)\left(b^{2} n_{2}^{2} \varepsilon_{2}+c^{2} n_{3}^{2} \varepsilon_{3}\right)}{R^{7}} \\
& R=\sqrt{a^{2} n_{1}^{2}+b^{2} n_{2}^{2}+c^{2} n_{3}^{2}}, \quad r_{1}=2 a^{2} n_{1}^{2}-b^{2} n_{2}^{2}-c^{2} n_{3}^{2}
\end{aligned}
$$

The dielectric susceptibility determined as a derivative of polarization with respect to a complete field $E_{1}=E_{01}+d_{1} K_{1}=E_{01} /\left(1-\alpha_{1} K_{1}\right)$ is

$$
\varepsilon_{1}(0, T, p)=\varepsilon_{1 \infty}^{a}+4 \pi \frac{\mu_{1}^{2}}{v} \frac{\tilde{\chi}_{1}}{1+\mu_{1}^{2} \tilde{\chi}_{1} K_{1}} .
$$

Let us mention that the difference between (6) and (12) is the larger the stronger the crystal lattice differs from a cubic one; at $a=b=c, K_{1} \equiv 0$.

Expressions analogous to (11)-(12) hold also for the dynamic transverse permittivity of antiferroelectrics [27].
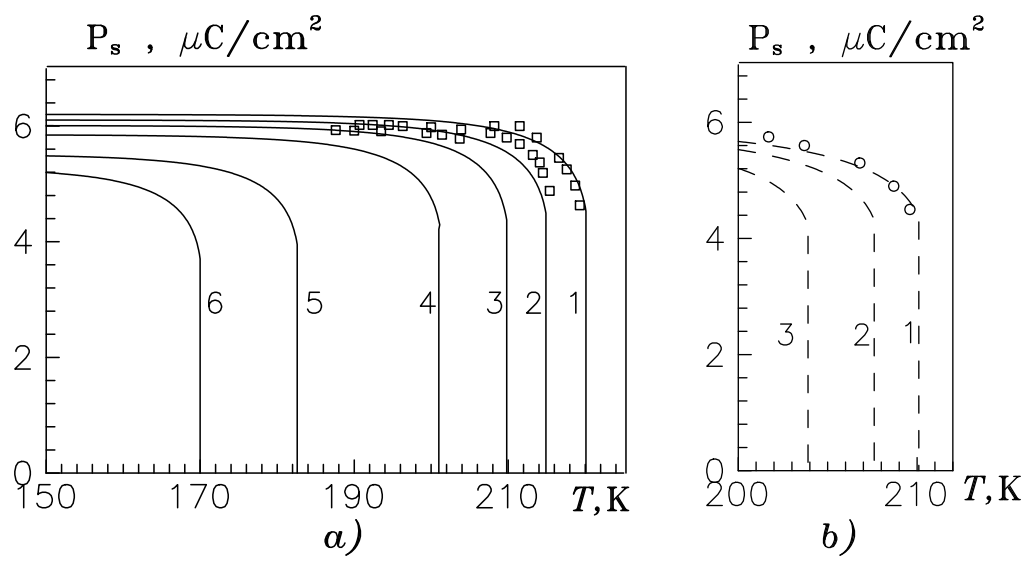

Figure 1. Temperature dependence of spontaneous polarization of $\mathrm{KD}_{2} \mathrm{PO}_{4}$ (a) and $\mathrm{K}\left(\mathrm{H}_{0.13} \mathrm{D}_{0.87}\right)_{2} \mathrm{PO}_{4}$ (b) crystals at different values of external pressure $p(\mathrm{kbar}):$ a) $\left(T_{\mathrm{C} 0}=220 \mathrm{~K}, \partial T_{\mathrm{C}} / \partial p=-2.5 \mathrm{~K} / \mathrm{kbar}\right) 1-0.001 ; 2-2.07 ; 3-$ $4.14 ; 4-7.6 ; 5-15.0 ; 6-20.0$. b) $\left(T_{\mathrm{C} 0}=210 \mathrm{~K}\right) 1-0.001 ; 2-0.2 ; 3-0.5$. Solid and dashed lines correspond to hydrostatic and uniaxial $p=-\sigma_{3}$ pressures, respectively. Experimental points are taken from [11] $-\square$ and [28] -0 .

In figure 1 we plot the temperature curves of spontaneous polarization of partially deuterated $\mathrm{K}\left(\mathrm{H}_{1-x} \mathrm{D}_{x}\right)_{2} \mathrm{PO}_{4}$ crystals at different values of hydrostatic and uniaxial $p=-\sigma_{3}$ pressures. As one can see, the model pressure dependence (9) of the effective dipole moment $\mu_{3}$ provides a satisfactory description of a decrease in saturation polarization with hydrostatic pressure. The theory also predicts a decrease with pressure of the jump of polarization at the transition point. That is due to a strong variation with pressure of the Slater energy $w$ (via (3)).

Since we accepted a negative value of $\delta_{1} / \delta_{0}$ for an uniaxial pressure $p=-\sigma_{3}$, then, according to (9), an effective dipole moment $\mu_{3}$ and thereby the spontaneous polarization is expected to decrease with this pressure. Unfortunately, no direct 


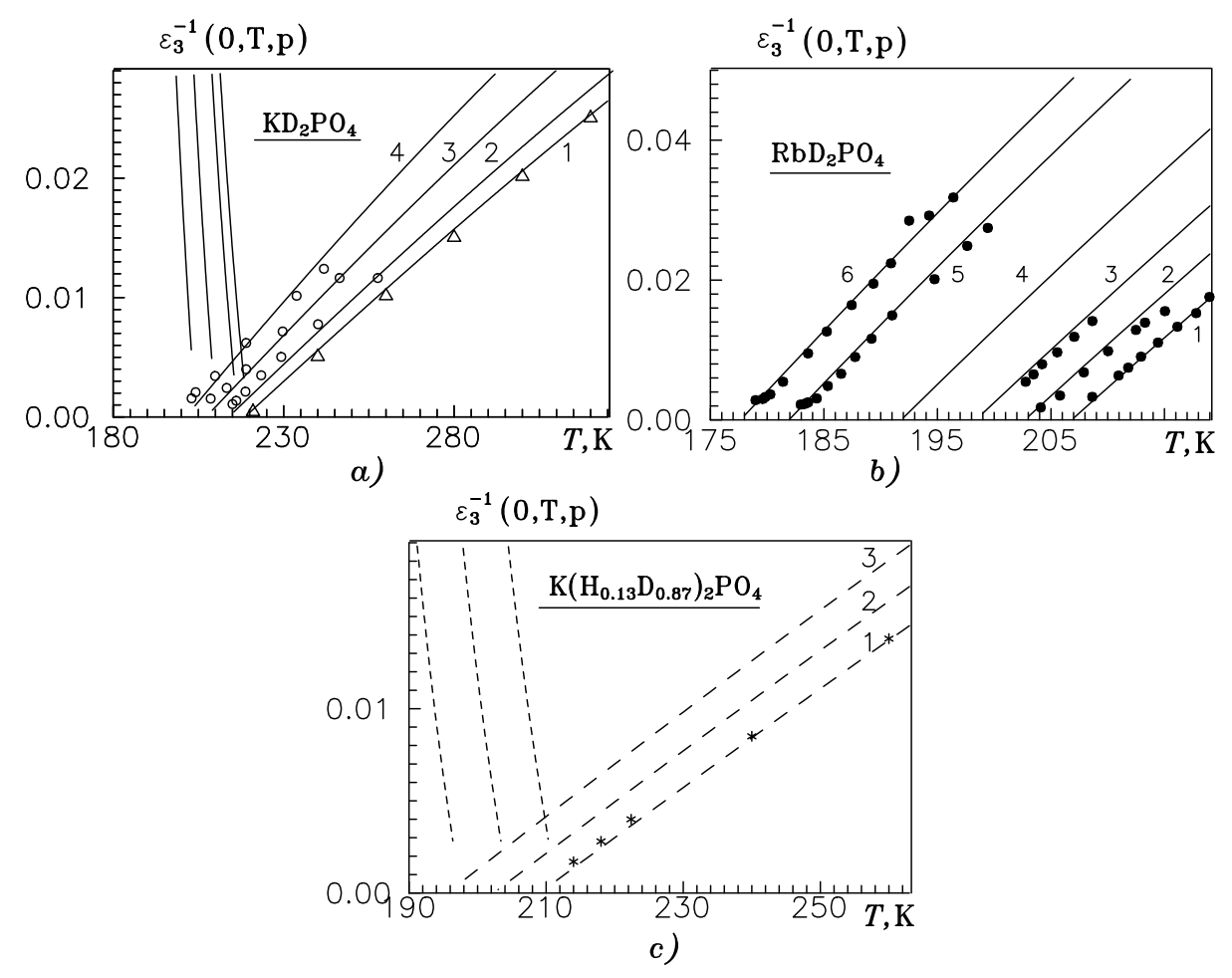

Figure 2. The temperature dependence of the inverse static longitudinal dielectric permittivity of $\mathrm{KD}_{2} \mathrm{PO}_{4}$ (a), $\mathrm{RbD}_{2} \mathrm{PO}_{4}(\mathrm{~b})$, and $\mathrm{K}\left(\mathrm{H}_{0.13} \mathrm{D}_{0.87}\right)_{2} \mathrm{PO}_{4}$ (c) crystals at different values of the external pressure $p$ (kbar): a) $\left(\partial T_{\mathrm{C}} / \partial p=-2 \mathrm{~K} / \mathrm{kbar}\right) 1$ $-0.001 ; 2-3.6 ; 3-4.7 ; 4-7.6$; b) $1-0.001 ; 2-1.1 ; 3-2.25 ; 4-4.0 ; 5-6.63 ; 6$ -7.76 ; c) $1-0.001 ; 2-0.5,3-1$. Experimental points are taken from: [29] $-\triangle$, $[12]-O,[14]-\bullet$, and [30] $-*$. Solid and dashed lines correspond to hydrostatic and uniaxial $p=-\sigma_{3}$ pressures, respectively.

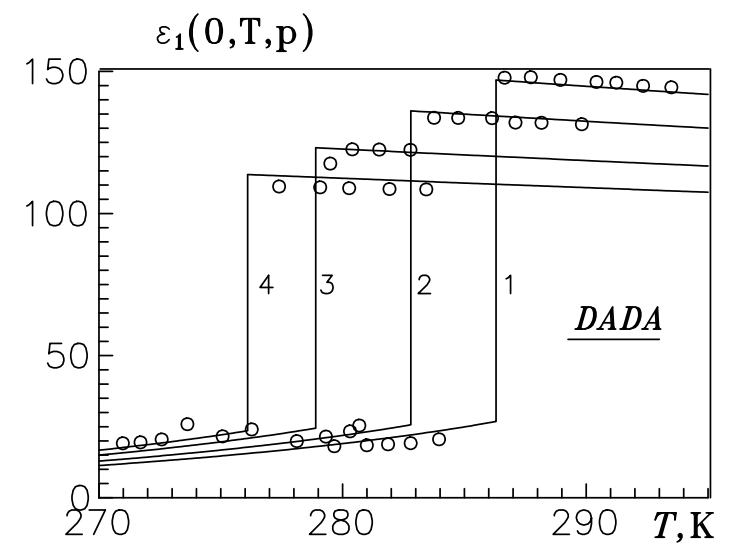

Figure 3. Temperature dependence of the transverse static dielectric permittivity of $\mathrm{ND}_{4} \mathrm{D}_{2} \mathrm{AsO}_{4}$ at different values of hydrostatic pressure $p$ (kbar): a) $1-0.001$; $2-2.62 ; 3-5.6 ; 4-7.68$. Experimental points are taken from [16]. 
experimental data for the uniaxial pressure $p=-\sigma_{3}$ on the spontaneous polarization of $\mathrm{KD}_{2} \mathrm{PO}_{4}$ are available.

In figure 2 we present the temperature dependences of the inverse static dielectric permittivity of $\mathrm{KD}_{2} \mathrm{PO}_{4}$ and $\mathrm{RbD}_{2} \mathrm{PO}_{4}$ crystals at different values of hydrostatic pressure and of $\mathrm{K}\left(\mathrm{H}_{0.13} \mathrm{D}_{0.87}\right)_{2} \mathrm{PO}_{4}$ at different $p=-\sigma_{3}$ pressures. The difference between permittivities of clamped and free crystals is neglected. Unfortunately, experimental data for non-zero pressures are available only for the hydrostatic pressure $[12,29]$. As one can see, the model dependences (9) provide a good description of the decrease in the Curie constant with hydrostatic pressure. Due to the adopted negative value of $\delta_{1} / \delta_{0}$, uniaxial pressure $p=-\sigma_{3}$ is expected to lower the Curie constant as well.
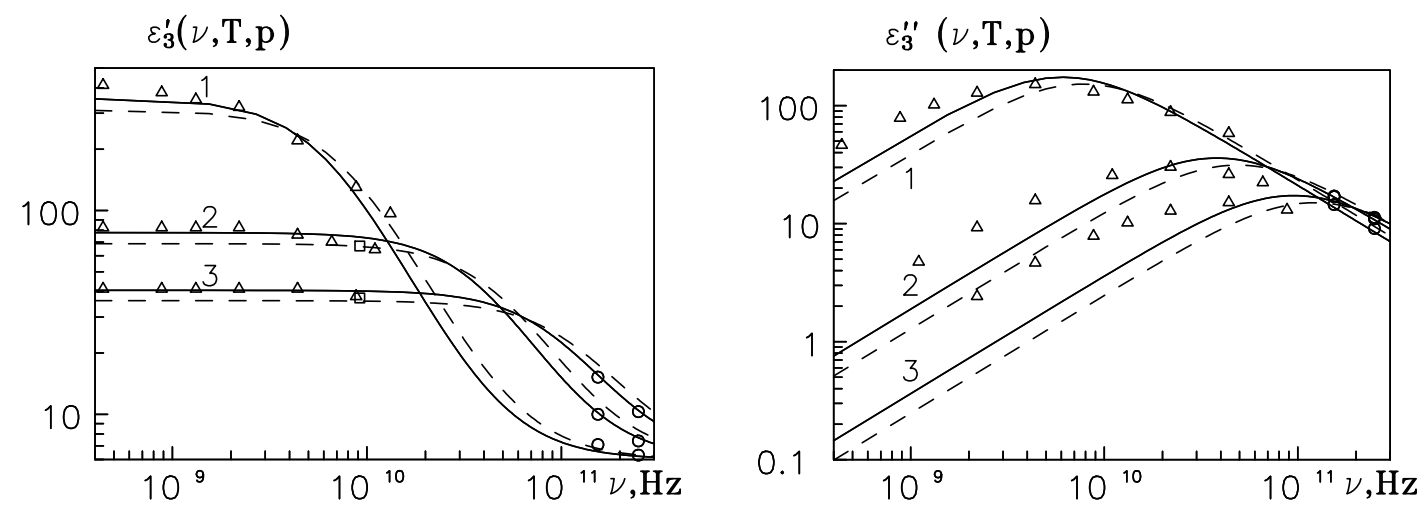

Figure 4. The frequency dependence of real and imaginary parts of longitudinal dynamic dielectric permittivity of $\mathrm{KD}_{2} \mathrm{PO}_{4}$ crystal at different temperatures $\Delta T(\mathrm{~K}): 1-10 ; 2-50 ; 3-100$ and hydrostatic pressures $p$ (kbar): solid lines 0.001 ; dashed lines -10 . Experimental points taken from $\triangle-[29], \square-[33], \bigcirc_{-}$ [34] correspond to atmospheric pressure.

Hence, similar to the pressure dependence of the transition temperature, the pressure dependences of dielectric permittivity and spontaneous polarization of these ferroelectrics are also governed by the parameter $\delta_{1} / \delta_{0}$, indicating the crucial role of the D-site distance in the dielectric response of the hydrogen bonded crystals.

In figure 3 we plot the temperature curves of the transverse dielectric permittivity of $\mathrm{ND}_{4} \mathrm{D}_{2} \mathrm{AsO}_{4}$ (DADA) at different values of hydrostatic pressure along with the experimental points by Gesi [16]. In the calculations, we use $\nu_{a}^{0}(0)=-35 \mathrm{~K}$ and value of the piezomodule $d_{14}$ corresponding to an undeuterated sample [31].

The pressure dependence of the coefficient $\mu_{1}^{2}$ calculated with (11) provides a fair description of a decrease in $\varepsilon_{1}^{a}$ in the paraelectric phase as well as of a slow increase in the antiferroelectric phase, showing thereby the importance of the mutual polarization mechanisms in the dielectric response of these crystals. It should be noted that there can also be other mechanisms of pressure effect upon the dipole moments of hydrogen bonded crystals, being neglected here: rotation of $\mathrm{PO}_{4}$ tetrahedra around the $c$-axis, shortening of $\mathrm{N}-\mathrm{H}-\mathrm{O}$ bonds in antiferroelectrics, etc. It can also be important that the dielectric permittivity of DADA [16] is measured at $\nu=10^{5} \mathrm{~Hz}$, 

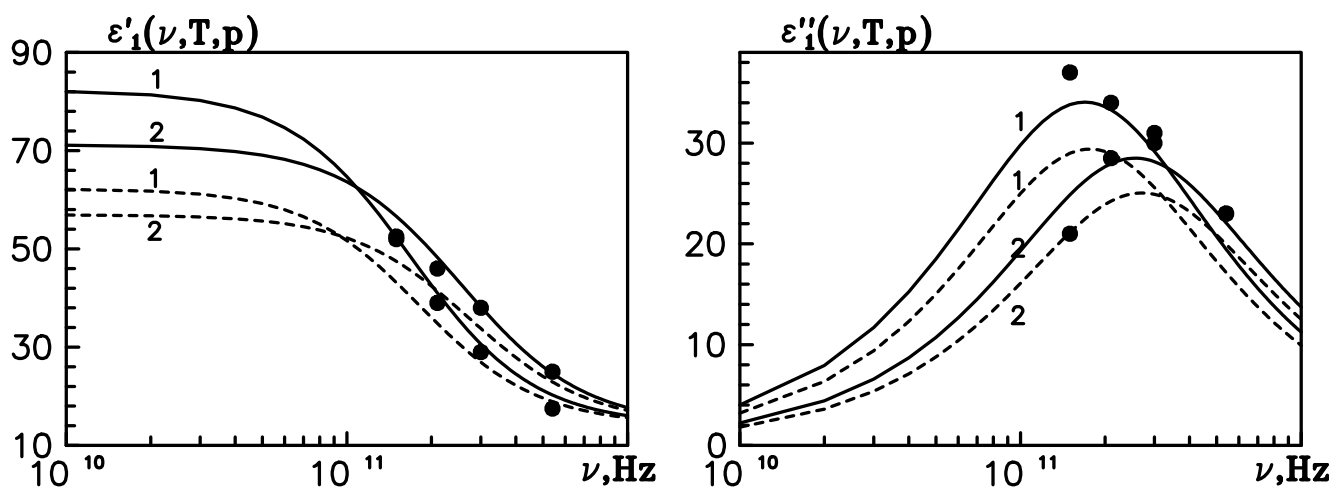

Figure 5. The frequency dependence of real and imaginary parts of transverse dynamic dielectric permittivity of $\mathrm{ND}_{4} \mathrm{D}_{2} \mathrm{PO}_{4}$ crystal at different temperatures $\Delta T(\mathrm{~K})$ : (a) $1-14,2-62$; (b) $1-18,2-64$ and hydrostatic pressures $p$ (kbar): solid lines - 0.001; dashed lines - 10. Experimental points taken from [32] correspond to atmospheric pressure.

which belongs to the region of the piezoelectric resonance.

No experimental data for the pressure effect upon the dynamic dielectric characteristics of the considered crystals is available. Predictions of our theory concerning the possible hydrostatic pressure effects on the dynamic dielectric permittivities of the strained hydrogen bonded crystals are illustrated in figures 4 and 5, where the theoretical frequency dependences of the longitudinal permittivity of $\mathrm{KD}_{2} \mathrm{PO}_{4}$ and transverse permittivity of $\mathrm{ND}_{4} \mathrm{D}_{2} \mathrm{PO}_{4}$ are plotted.

Numerical calculations show that the main contributions into the $\varepsilon_{3}(\nu, T, p)$ in $\mathrm{KD}_{2} \mathrm{PO}_{4}$ and $\varepsilon_{1}(\nu, T, p)$ in $\mathrm{ND}_{4} \mathrm{D}_{2} \mathrm{PO}_{4}$ are going from the first relaxational modes $\left(\chi_{1} \gg \chi_{2}, \tau_{1} \gg \tau_{2}\right)$, i.e. the dispersion is of Debye type. The experimental data for $\varepsilon_{3}^{\prime}(\nu, T, p)$ and $\varepsilon_{1}^{\prime}(\nu, T, p)$ for the ambient pressure case are rather well described by the theory. The agreement between the theory and the experiment for $\varepsilon^{\prime \prime}$ at frequencies lower than the dispersion frequency is much worse, though the character of the dependence is qualitatively reproduced.

Pressure dependences of the effective dipole moments $\mu_{3}$ for $\mathrm{KD}_{2} \mathrm{PO}_{4}$ and $\mu_{1}$ for $\mathrm{ND}_{4} \mathrm{D}_{2} \mathrm{PO}_{4}$ were calculated from (9) and (11), respectively. The theory predicts that the external pressure does not affect the Debye character of the dielectric relaxation. Above the transition point at frequencies $10^{10} \div 10^{12} \mathrm{~Hz}$ both real and imaginary parts of the transverse dielectric permittivity of $\mathrm{ND}_{4} \mathrm{D}_{2} \mathrm{PO}_{4}$ should decrease with pressure, whereas those of the longitudinal permittivity of $\mathrm{KD}_{2} \mathrm{PO}_{4}$ decrease with pressure at frequencies below the dispersion frequency and increase above it.

\section{Concluding remarks}

Within the presented approach we describe the effects of hydrostatic and uniaxial pressures on the static and dynamic dielectric properties of deuterated ferroelectrics and antiferroelectrics of KDP family.

The main parameter which determines the pressure dependences of the sponta- 
neous polarization and static dielectric permittivities is the ratio $\delta_{1} / \delta_{0}$, which is the rate of the pressure-induced changes in the $\mathrm{D}$-site distance. The suggested model pressure dependences of the effective dipole moments of unit cells provide a satisfactory agreement with the experimental data for the effects of hydrostatic pressure on the static dielectric properties of the crystals. We show that for the antiferroelectric crystals of the family with large values of transverse dipole moments, one should take into account the processes of mutual polarization of unit cell dipoles. For the ferroelectrics with small longitudinal moments those processes are not important.

Dielectric relaxation in the strained hydrogen bonded crystals is studied. Possible pressure dependences of the dynamic dielectric permittivities of $\mathrm{KD}_{2} \mathrm{PO}_{4}$ and $\mathrm{ND}_{4} \mathrm{D}_{2} \mathrm{PO}_{4}$ crystals are presented.

Further dielectric and structural measurements of the pressure effects, especially of uniaxial pressures, on the KDP family crystals will allow us to ascertain the values of the theory parameters, to verify its predictions regarding the possible changes in the H-bond geometry and dielectric properties of the crystals.

\section{Acknowledgements}

Authors would like to thank participants of the IV Ukrainian-Polish Meeting on Phase Transitions and Ferroelectrics Physics (Dnipropetrovsk, Ukraine, June 1998) for their interest to the work and valuable discussions.

This work was supported by the Foundation for Fundamental Investigations of the Ukrainian Ministry in Affairs of Science and Technology, project No 2.04/171.

\section{References}

1. Tominaga Y. // Ferroelectrics, 1983, vol. 52, p. 91-100.

2. Tokunaga M., Tatsuzaki I. // Phase Trans., 1984, vol. 4, p. 97-156.

3. Piltz R.O., McMahon M.I., Nelmes R.J. // Ferroelectrics, 1990, vol. 108, p. 271-276.

4. Stasyuk I.V., Levitskii R.R., Moina A.P. (to be published in Phys. Rev. B.)

5. Levitskii R.R., Moina A.P. Preprint of the Institute for Condensed Matter Physics, ICMP-98-19E, Lviv, 1998, 24 p.

6. Levitskii R.R., Moina A.P., Zachek I.R. // (submitted to Cond. Matt. Phys.).

7. Blinc R., Žekš B. Soft modes in ferroelectrics and antiferroelectrics. New York, Elseviers, 1974.

8. Samara G.A. // Ferroelectrics, 1987, vol. 71, p. 161-182.

9. Blinc R, Žekš B. // Helv. Phys. Acta, 1968, vol. 41, p. 701-706.

10. Torstveit S. // Phys.Rev.B, 1979, vol. 20 , No. 11, p. 4431-4441.

11. Samara G.A. // Ferroelectrics, 1979, vol. 22, p. 925-936.

12. Zhukov S.G., Kul'bachinskii V.A., Smirnov P.S., Strukov B.A., Chudinov S.H. // Bull. Ac.Sci.USSR. Phys.Ser., 1985, vol. 49, No. 2, p. 40-42.

13. Hikita T., Ono Y., Bungo A. // J. Phys. Soc. Jap., 1992, vol. 61, No. 10, p. 3794-3798.

14. Gesi K., Ozawa K., Osaka T., Makita Y. // J. Phys. Soc. Jap., 1992, vol. 61, No. 1, p.342-347.

15. Spillman W.B., Leung R.C., Tornberg N.E., Lowndes R.P. // Ferroelectrics, 1977, vol. 17, p. 383-385. 
16. Gesi K., Ozawa K. // J. Phys. Soc. Jap., 1984, vol. 53, No. 12, p. 4405-4412.

17. Stadnik N.I., Romanyuk N.A., Chervonyj R.G. // Opt. Spectrosk. 1998, vol. 84, p.273.

18. Nelmes R.J. // Ferroelectrics, 1987, vol. 71, p. 87-123.

19. Tibbals J.E., Nelmes R.J., McIntyre G.J. // J.Phys.C: Solid State. Phys., 1982, vol. 15 , p. $37-58$.

20. Levitskii R.R., Zachek I.R., Moina A.P., Duda A.S., Shcherbina Ye.V. // (Submitted to Functional Materials).

21. Levitskii R., Zachek I., Mits Ye., Moina A. // Fiz. Zbirnyk NTSH, 1998, vol. 3, p. 417446 (in Ukrainian).

22. Levitskii R.R., Moina A.P. // Cond. Matt. Phys., 1998. vol. 1, No. 2, p. 365-382.

23. Havlin S., Litov E., Sompolinsky H. // Phys. Rev. B, 1976, vol. 14, No. 3, p. 12971302.

24. Glauber J. Time-dependent statistics of the Ising model. // J. Math. Phys., 1963, vol. 4, No. 2, p. 294-307.

25. Känzig W. Ferroelectrics and Antiferroelectrics. New York, Academic Press Inc., 1957.

26. Skalyo J., Jr., Frazer B.C., Shirane G., Daniels W.B. // J. Phys. Chem. Solids, 1969, vol. 30, p. 2045-2051.

27. Moina A.P. Doctoral thesis, Inst. for Cond. Matt. Phys., Lviv, 1997 (in Ukrainian).

28. Chabin M., Giletta E. // Ferroelectrics, 1977, vol. 15, p. 149-154.

29. Hill R.M., Ichiki S.K. // Phys.Rev., 1963, vol. 130, No. 1, p. 150-151.

30. Chabin M., Giletta G. // Phys. Stat. Sol(b), 1980, vol. 100, p. K77-K82.

31. Adhav R.S. // J. Acoust. Soc. Am., 1968, vol. 43, No 4, p. 835-838.

32. Kozlov V.G., Lebedev S.P., Prokhorov A.M., Volkov A.A. // J. Phys. Soc. Japan, 1980, vol. 49, Suppl., p. 188-190.

33. Kaminov I.P. // Phys. Rev., 1965, vol. 138, No. 5A, p. 1539-1543.

34. Volkov A.A., Kozlov G.V., Lebedev S.P. // Fiz. Tv. Tela, 1979, vol. 21, p. 3304 (in Russian).

\title{
Вплив зовнішнього тиску на діелектричний відгук кристалів сім'ї KDP
}

\author{
І.В.Стасюк, Р.Р.Левицький, А.П.Моїна \\ Інститут фізики конденсованих систем НАН України, \\ 79011 Львів, вул. Свєнціцького, 1
}

Отримано 14 січня 1999 р.

В наближенні чотиричастинкового кластера для моделі протонного впорядкування досліджуються ефекти, викликані впливом зовнішніх тисків, що не понижують симетрії кристалів, на статичні і динамічні діелектричні властивості дейтерованих сегнетоелектриків і антисегнетоелектриків сім'ї $\mathrm{KH}_{2} \mathrm{PO}_{4}$. Теорія забезпечує задовільний опис наявних експериментальних даних. Показано важливість індукованої тиском зміни віддалі між можливими положеннями дейтрона на зв'язку у діелектричному відгуку кристалів з водневими зв'язками.

Ключові слова: KDP, тиск, спонтанна поляризація, діелектрична проникність

PACS: $77.84 . F a$ 